\title{
Foreign Body Reaction after Implantation of a Device for Intervertebral Assisted Motion
}

\author{
Jun-Yeong Seo, M.D., ${ }^{1}$ Kee-Yong Ha, M.D., Ph.D., ${ }^{2}$ Young-Hoon Kim, M.D., ${ }^{2}$ Joo-Hyun Ahn, M.D. ${ }^{2}$ \\ Department of Orthopaedic Surgery, ${ }^{1}$ Jeju National University Hospital, School of Medicine, Jeju National University, Jeju, Korea \\ Department of Orthopaedic Surgery, ${ }^{2}$ Seoul St. Mary's Hospital, College of Medicine, The Catholic University of Korea, Seoul, Korea
}

\begin{abstract}
The device for intervertebral assisted motion (DIAM) is a dynamic implant that consists of a silicone bumper enveloped by a polyethylene terephthalate (PET) fiber sack. Silicone and PET were used because of their biological inertness, but repetitive motion of the spine can cause wear on the implant nonetheless. The purpose of this study is to report a case of foreign body reaction (FBR) against a DIAM. A 72-year-old female patient presented with lower back pain and both legs radiating pain. She had undergone DIAM implantation at $L 4-5$ for spinal stenosis 5 years previously. The intervertebral disc space of L4-5, where the DIAM was inserted, had collapsed and degenerative scoliosis had developed due to left-side collapse. MRI showed L3-4 thecal sac compression and left L4-5 foraminal stenosis. The patient underwent removal of the DIAM and instrumented fusion from L3 to L5. During surgery, fluid and granulation tissue were evident around the DIAM. Histopathology showed scattered wear debris from the DIAM causing chronic inflammation due to the resulting FBR. A FBR due to wear debris of a DIAM can induce a hypersensitivity reaction and bone resorption around the implant, causing it to loosen.
\end{abstract}

Key Words : Device for intervertebral assisted motion $\cdot$ Foreign body reaction $\cdot$ Polyethylene terephthalate $\cdot$ Silicone.

\section{INTRODUCTION}

Recently developed motion-preserving spinal implants that consist of new synthetic materials, including disc replacements and dynamic posterior stabilization systems, may cause a foreign body reaction (FBR) around the implant itself or due to unintended wear debris. Wear and tear on the implant may occur by repetitive spinal motion in daily life, which can induce a FBR against the wear particles. When this condition becomes chronic, local osseous and epidural soft-tissue structures can be affected $^{4)}$. In addition, a FBR and fibrous encapsulation develop around the implant even in the absence of wear particles by a series of inflammatory and wound healing processes ${ }^{2}$. Here, we present a case of FBR causing loosening of a device for intervertebral assisted motion (DIAM; Medtronic Spine and Biologics, Memphis, TN, USA) 5 years after implantation. To the best of the author's knowledge, a FBR due to polyethylene wear after implantation of a DIAM has been reported only once previously ${ }^{7)}$.

\section{CASE REPORT}

A 72-year-old female visited our hospital for intractable lower back pain that had radiated into her legs for 1 month. She had undergone decompressive laminotomy and DIAM insertion at L4-5 for spinal stenosis 5 years previously. Two years later, she underwent decompressive laminotomy at L3-4 for newly developed spinal stenosis at the adjacent segments. Plain lateral radiographs showed degenerative spondylolisthesis of L3-4 and collapse of the intervertebral disc space of L4 -5 , as compared to the initial plain radiographs. Plain anteroposterior radiographs showed scoliotic changes due to left sided collapse of the L4-5 disc space, which were not seen on immediate postoperative radiographs (Fig. 1). MRI showed L3-4 thecal sac compression and left L4 -5 foraminal stenosis. High signal changes indicating fluid collection around the implanted DIAM were also seen (Fig. 2). Laboratory tests revealed an elevated erythrocyte sedimentation rate of $65 \mathrm{~mm} / \mathrm{h}$ but a normal C-reactive protein level of $0.08 \mathrm{mg} / \mathrm{dL}$. The white blood cell count was $5.05 \times 10^{9} / \mathrm{L}$.

The patient underwent removal of the DIAM system and instrumented fusion at L3-5. Surgical exploration revealed that

- Received : June 9, $2014 \cdot$ Revised : September 25, $2014 \cdot$ Accepted : October 22, 2014

- Address for reprints : Kee-Yong Ha, M.D., Ph.D.

Department of Orthopaedic Surgery, Seoul St. Mary's Hospital, College of Medicine, The Catholic University of Korea, 222 Banpo-daero, Seocho-gu, Seoul 06591, Korea

Tel : +82-2-2258-2837, Fax : +82-2-535-9834, E-mail : kyh@catholic.ac.kr

- This is an Open Access article distributed under the terms of the Creative Commons Attribution Non-Commercial License (http://creativecommons.org/licenses/by-nc/3.0) which permits unrestricted non-commercial use, distribution, and reproduction in any medium, provided the original work is properly cited. 


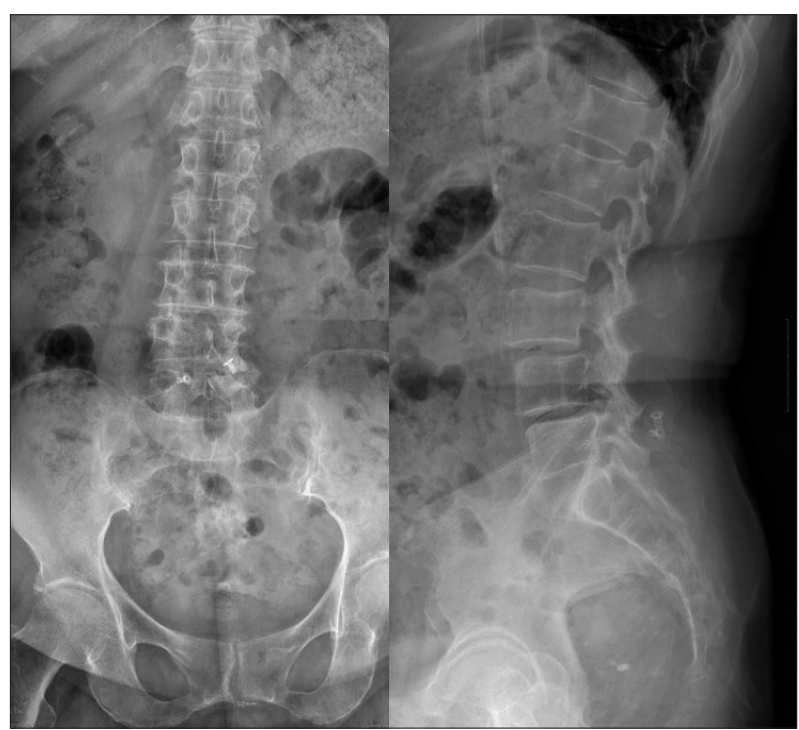

Fig. 1. Post-operative plain radiographs taken 5 years after DIAM insertion showed marked disc space collapse and scoliosis caused by leftside disc space collapse at $L 4-5$, as compared to the immediate postoperative radiographs. Spondylolisthesis of L3 and L4 was also found. DIAM : device for intervertebral assisted motion.

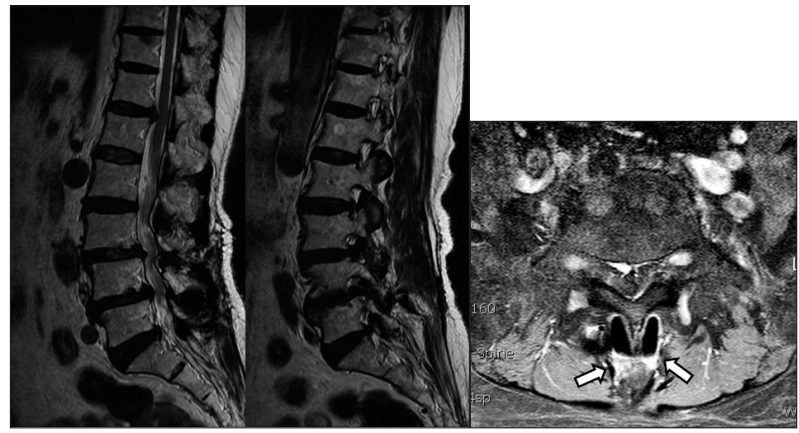

Fig. 2. MRI showed L3-4 thecal sac compression and left L4-5 foraminal stenosis. Increased signal change indicating fluid collection around the implanted DIAM was evident (white arrows). DIAM : device for intervertebral assisted motion.

the DIAM had loosened around the inflammatory granulation tissues and was surrounded by scant serous fluid. The soft tissues around the DIAM were sampled for pathology. Histopathology showed numerous wear particles scattered with chronic inflammatory cells and foreign body giant cells (FBGCs) (Fig. 3). Bacterial cultures of the biopsies were negative.

Her back pain improved after the L3-5 fusion surgery. The visual analogue scale score of her back pain improved from 10 preoperatively to 4 at 2 weeks postoperatively and to 1 at 2 years postoperatively.

\section{DISCUSSION}

The DIAM is an interspinous distraction device that consists of an X-shaped silicone (dimethylsiloxane) interspinous process bumper enveloped by a polyethylene terephthalate (PET) fiber $\operatorname{sack}^{8)}$. It acts as a dynamic stabilizer that preserves motion and
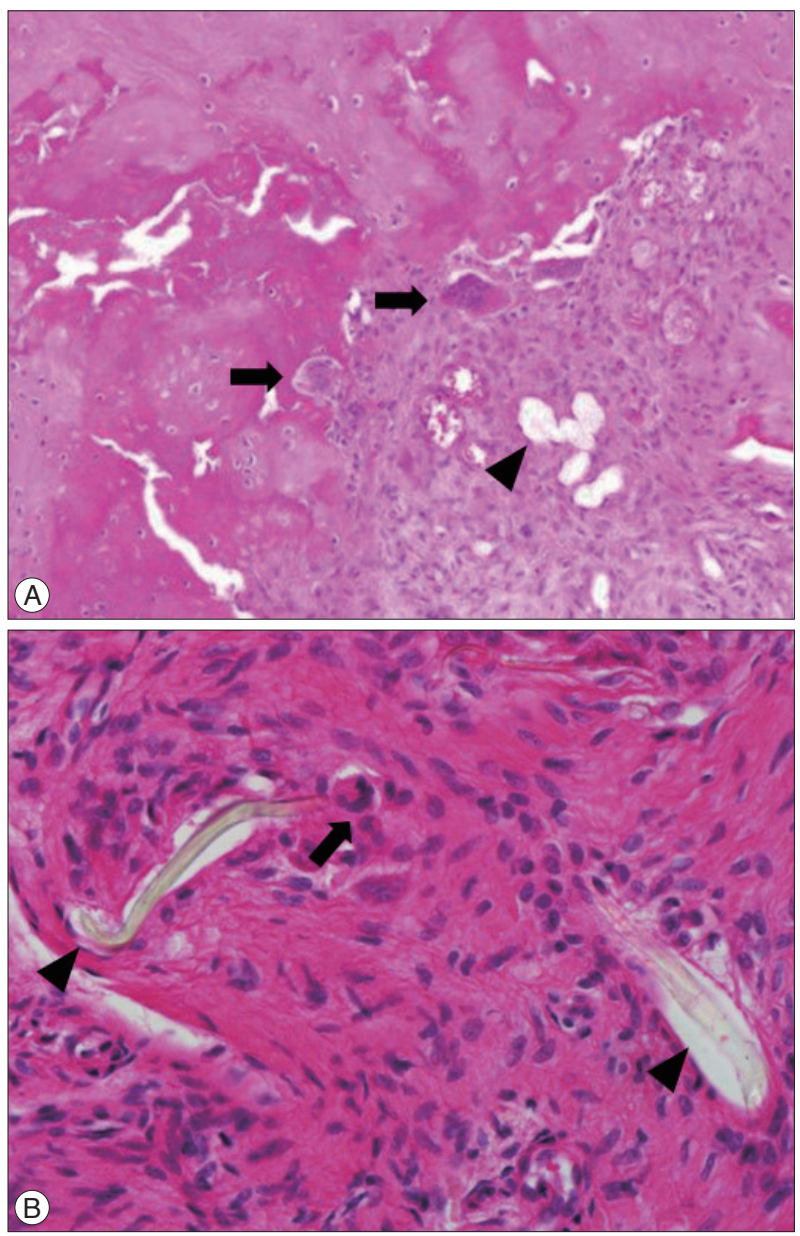

Fig. 3. A : Pathologic findings showing infiltration of polymorphous inflammatory cells and foreign-body-type giant cells (arrows) around the foreign bodies (arrow head). B : Fibrils (arrow heads) from the polyethylene terephthalate fibers sacs of the DIAM. Inflammatory cells, and foreign body type giant cells (arrows) were shown [Hematoxylin-eosin; (A) $\times 100$, (B) $\times 400)$ ]. DIAM : device for intervertebral assisted motion.

provides segmental stabilization. However, Ha et al. ${ }^{6}$ reported that the DIAM had lost its indirect decompression by distraction that may be caused by loss of the elasticity of the components of the implant.

The allowed spinal motion paradoxically might stress the implant itself. Wear and tear can develop and eventually lead to long-term issues in plastic surgery, such as silicone granulomas". In this process, the PET or silicone that the DIAM is made from can produce wear debris that then leads to a FBR. In addition, foreign bodies can be introduced during the surgical procedure itself. The microarchitecture of the PET fiber sac can also be damaged, as one wing of the implant is squeezed and compressed during insertion between the interspinous processes.

Cunningham et al. ${ }^{4)}$ reported that during the epidural application of PET and silicone, the debris can elicit a chronic histiocytic reaction. In addition, an FBR can develop around the material itself, even in the absence of wear debris ${ }^{10}$. Macrophages and FBGCs are critical in the formation of an $\mathrm{FBR}^{3)}$. In our case, fibrils from the PET fiber sac were scattered in the tissue around the 
DIAM and acted as foreign bodies. Histiocytes, polymorphous inflammatory cells, and FBGCs had infiltrated the space around the implant. Polyethylene debris, as in total joint replacement, is taken up by macrophage giant cells that release prostaglandin $\mathrm{E} 2$, which resorbs bone, causing the implant to loosen and leading to a vicious cycle of wear and loosening ${ }^{5)}$.

The lumbar spine is subject to much external force. It supports the body weight and is a highly mobile region. An implanted DIAM also has to endure the stress of body weight and lumbar motion. Even if most implants induce FBR in some degree, lumbar motion and body weight can jeopardize the interspinous device, accelerating its wear and tear. The spinous process around the DIAM can be resorbed and the implant can loosen, eventually causing lower back pain and/or radiating pain ${ }^{1)}$. In this case, the back and radiating pain were assumed to be caused by implant loosening and segmental instability. Disc space collapse in both the coronal and sagittal planes might give rise to foraminal stenosis. Although we were unable to differentiate the origin of the lower back pain as from post-laminectomy instability or FBR, macrophages and FBGCs were demonstrated on histological examination. The patient improved markedly after removing most of the granulation tissue, profuse irrigation, and L3-4 fusion. We believe that the FBR contributed markedly to her symptoms preoperatively.

\section{CONCLUSION}

A FBR from wear debris of a DIAM can induce a hypersensitivity reaction and bone resorption around the implant, causing it to loosen. Therefore, surgeons should insert the DIAM me- ticulously so as not to generate wear debris during the surgery. In patients with a DIAM who complain of back and radiating pain, a FBR must be considered, and the patients should be monitored long term.

\section{References}

1. Akhaddar A, Boulahroud O, Naama O, Al-Bouzidi A, Boucetta M : Paraspinal textiloma after posterior lumbar surgery : a wolf in sheep's clothing. World Neurosurg 77 : 375-380, 2012

2. Anderson JM : Inflammatory response to implants. ASAIO Trans 34 : 101-107, 1988

3. Anderson JM : Multinucleated giant cells. Curr Opin Hematol 7 : 4047, 2000

4. Cunningham BW, Hallab NJ, Hu N, McAfee PC : Epidural application of spinal instrumentation particulate wear debris : a comprehensive evaluation of neurotoxicity using an in vivo animal model. J Neurosurg Spine $19: 336-350,2013$

5. Daniel J, Holland J, Quigley L, Sprague S, Bhandari M : Pseudotumors associated with total hip arthroplasty. J Bone Joint Surg Am 94 : 86-93, 2012

6. Ha KY, Seo JY, Kwon SE, Son IN, Kim KW, Kim YH : Posterior dynamic stabilization in the treatment of degenerative lumbar stenosis : validity of its rationale. J Neurosurg Spine 18 : 24-31, 2013

7. Jerosch J, Moursi MG : Foreign body reaction due to polyethylene's wear after implantation of an interspinal segment. Arch Orthop Trauma Surg $128: 1-4,2008$

8. Kim DH, Albert TJ : Interspinous process spacers. J Am Acad Orthop Surg 15 : 200-207, 2007

9. Potter EH, Rohrich RJ, Bolden KM : The role of silicone granulomas in recurrent capsular contracture : a review of the literature and an approach to management. Plast Reconstr Surg 131 : 888e-895e, 2013

10. Rodriguez A, Meyerson H, Anderson JM : Quantitative in vivo cytokine analysis at synthetic biomaterial implant sites. J Biomed Mater Res A 89 : 152-159, 2009 